\title{
Research on Chaos Electromagnetic Mechanism Fusion Algorithm Based on Logistic Mapping
}

\author{
Xunlai $\mathrm{He}^{1}$, Junhui Yin ${ }^{2, *}$, Weizhao Zhang ${ }^{2}$ and Zhenqian Yang ${ }^{3}$ \\ ${ }^{1}$ Nantong Institute of Technology, Jiangsu China \\ ${ }^{2}$ Shijiazhuang campus of Army Engineering University, Hebei China \\ ${ }^{3}$ Xi' an Satellite Measurement and Control Center, Shanxi China \\ ${ }^{*}$ Corresponding author
}

\begin{abstract}
For multi-component, strong nonlinear systems, especially when considering component elasticity and gaps, it is difficult to obtain high-order information such as required gradients through calculations. It will face problems such as fewer iterations and inconspicuous optimization results, and because of the objective function. The non-linear features make it difficult to achieve optimal calculations. In this paper, by introducing Logistic chaotic maps to enrich the search behavior, the global optimization ability of the standard electromagnetic algorithm is improved. The test results show that the improved EM algorithm introduced with the chaos mechanism, the CEM algorithm, improves both the accuracy of the solution and the convergence efficiency. For solving high-dimensional function optimization problems. At the same time, it is found that the better initial population is of great significance for speeding up the optimization process, and the initial fitness can be improved by adopting multiple initial population selection and setting elite solutions.
\end{abstract}

Keywords—nonlinear systems; logistic mapping; EM algorithm; multi-objective optimization; chaos local search; charged particles

\section{INTRODUCTION}

In recent years, in the field of mechanical system design, improvement and optimization, the multi-objective linear weighting method based on the single-objective optimization strategy and the Pareto optimal theory have been widely used to solve many practical engineering problems. However, there are often complex relationships that are difficult to determine between the various indicators of the system. If weights are simply weighted to construct an optimized objective function, there is a clear subjectivity and it is not conducive to obtaining scientific and reasonable optimization results. The analysis believes that the limitation of the above method is rooted in the fact that only the optimization goal is located in the optimization of the system comprehensive index, which causes the objective function to basically depend on the subjective choice of the weight of each index.

As the scale and complexity of optimization problems continue to increase, it is becoming more and more difficult to solve problems using a single optimization algorithm [1]. It is sometimes difficult to achieve good results simply by relying on the improvement of individual indicators in the algorithm. The application of excellent algorithms to integrate, using their complementary advantages to solve problems together will be a good idea.

Therefore, this paper establishes a Chaos Electromagnetic Mechanism Fusion Algorithm (CEM), which improves the global optimization ability of EM algorithm by introducing Logistic chaotic map.

\section{EleCtromagnetic Mechanism Algorithms AND CORRECTION METHODS}

Birbil S.I. and Shu-cherng Fang [2] proposed a novel heuristic-based optimization method based on the attraction-repulsion mechanism among charged particles in electromagnetic fields in the field of artificial intelligence and optimization-Electromagnetism-like mechanism (EM). The algorithm and its basic principles and mathematical models were systematically described [3]. The current results show that the EM algorithm can solve the problem of function optimization [4]. It has the advantages of less control parameters, flexible structure, and strong compatibility with other algorithms, and the convergence has been proved theoretically. However, the standard algorithm also has the same premature problem. To better realize the global optimization, the EM algorithm should be properly improved in combination with the actual research.

To establish a nonlinear unconstrained optimization problem with bounded variables so that the EM algorithm can be described

$$
\begin{aligned}
\min & f(X) \\
\text { s. } t . & x \in S
\end{aligned}
$$

In formula (1), $f: R^{\mathrm{n}} \rightarrow R$ is nonlinear functions, $n$ is dimension of the problem, $S$ is a bounded feasible domain, $u_{k}$ and $l_{k}$ are the upper and lower bounds of the k-th dimension, $f(X)$ is the minimization function to be found.

\section{Step 1, Population initialization}

If the NO. $k$ dimensional component of the charged 
particle $X_{i}$ is represented by $X_{i}^{k}$, the population is generated as follows

$$
X_{i}^{k}=l+\operatorname{rand}(0,1) \cdot\left(u_{k}-l_{k}\right)
$$

Calculate the objective function value of each charged particle and record the optimal particle as $X_{\text {best }}$.

\section{Step 2, EM local search}

Local search is performed on a single charged particle to improve the optimal solution that the population has searched for each charged particle is searched within a specified number of times in each dimension according to a certain step size. Once a better solution is obtained, the search for this dimension is stopped.

\section{Step 3, Combine force calculation}

The magnitude of the attraction or repulsion force of a charged particle $X_{i}$ is determined by its amount of charge $Q_{i}$. The formula is

$$
Q_{i}=\exp \left(-n \frac{f\left(X_{i}\right)-f\left(X_{\text {best }}\right)}{\sum_{i=1}^{m}\left(f\left(X_{i}\right)-f\left(X_{\text {best }}\right)\right)}\right), \forall i
$$

In formula (3), particles with better objective function values will have a larger charge and a stronger attraction. Different from the concept of charge in physics, the charge of each particle in the formula is not positive or negative, and the direction of the force between two particles can be determined after comparing the objective function values.

When $i \neq j$, the electromagnetic force of the charged particles $X_{i}$ subjected to the particles $X_{j}$ is

$F_{i, j}=\left\{\begin{array}{ll}\left(X_{j}-X_{i}\right) \frac{Q_{i} \cdot Q_{j}}{\left\|X_{j}-X_{i}\right\|^{2}}, & f\left(X_{j}\right) \leq f\left(X_{i}\right) \\ \left(X_{i}-X_{j}\right) \frac{Q_{i} \cdot Q_{j}}{\left\|X_{j}-X_{i}\right\|^{2}}, & f\left(X_{j}\right)>f\left(X_{i}\right)\end{array}, i, j=1, \cdots, m\right.$

From equation (4), it can be seen that charged particles with smaller target function values will attract poorer particles, while particles with larger target function values will reject better particles due to the current optimal particle $X_{\text {best }}$ has the smallest objective function value, so it acts as an absolute attractor and exerts a strong attraction to all other particles in the population.

After calculating the electromagnetic force exerted by the other $m-1$ charged particles on $X_{i}$, the force of particle $X_{i}$ can be vector superposed according to equation (5).

$$
F_{i}=\sum_{j=1}^{m} F_{i, j}, \quad j \neq i
$$

\section{Step 4, Individual moves}

After obtaining the electromagnetic resultant vector $F_{i}$ of each charged particle $X_{i}, X_{i}$ will move in the direction of the resultant force by a random step size of $\lambda=\operatorname{rand}(0,1)$, and its movement equation is

$$
X_{i}=X_{i}+\operatorname{rand}(0,1) \cdot \frac{F_{i}}{\left\|F_{i}\right\|} \cdot R N G
$$

In equation (6), $R N G$ is a vector in which each component defines a corresponding feasible step that moves toward the upper boundary $u_{k}$ or the lower boundary $l_{k}$,

$$
R N G= \begin{cases}u_{k}-X_{i}^{k}, & F_{i}^{k}>0 \\ X_{i}^{k}-l_{k}, & F_{i}^{k} \leq 0\end{cases}
$$

In equation (7), $F_{i}^{k}$ is the $k$ dimensional component of the resultant force $F_{i}$.

This completes an iteration of the EM algorithm, and the location of each charged particle has been updated [5]. 2)

Step 5, Termination criteria (if not satisfied, go to step

As a heuristic optimization method, EM algorithm mainly adopts two types of termination criteria:

1. When the number of iterations reaches a preset limit, the algorithm terminates. For moderate difficulty functions, it is generally converged to an optimum after 25 iterations per dimension.

2. The current optimal particle still does not change after a certain number of iterations. That is, when the minimum error criterion is reached, the algorithm terminates. If only this criterion is used, it is possible to prematurely terminate the algorithm before it converges to global optimum.

3. In addition, there is a class of criteria where the algorithm ends when the value of the objective function is $\varepsilon$. However, this termination criterion is not feasible without knowing the global optimal solution in advance, so it is of little significance for practical engineering problems.

Based on the above analysis, the former two types of criteria are used in conjunction with the termination criterion, that is, the maximum number of iterations is set in advance (recorded 
as N1), and the algorithm terminates when the optimal particle fitness level reaches the minimum error criterion.

\section{Step 6, algorithm correction (prevent premature measures)}

In order to prevent the algorithm from falling into the local optimum prematurely, Birbil, Fang, and SHEU corrected the original EM algorithm and added a measure to prevent premature convergence, that is, to select a particle outside the current optimal particle $X_{\text {best }}$ and use the perturbation of the particle to make It moves to areas within the feasible region that are easily ignored by the algorithm, enhancing the algorithm's global search capabilities.

In the revised EM algorithm, the particle $X_{P}$ that is farthest from the current best particle $X_{\text {best }}$ in the population is taken as disturbing particle,

$$
X_{P}=\arg \max \left\{\left\|X_{P}-X_{i}\right\|, \quad i=1,2, \cdots, m\right\}
$$

When each charged particle is forced, the particles other than the perturbation particle $X_{P}$ still calculate the resultant force according to the equations (4) and (5), and the particle $X_{P}$ is calculated according to the following equation. In equation (9), $\mu \in U(0,1)$.

$$
F_{P}=\sum_{j \neq P}^{N}\left\{\begin{array}{l}
\left(X_{j}-X_{P}\right) \frac{\mu \cdot Q_{P} \cdot Q_{j}}{\left\|X_{j}-X_{P}\right\|^{2}}, f\left(X_{j}\right) \leq f\left(X_{P}\right) \\
\left(X_{P}-X_{j}\right) \frac{\mu \cdot Q_{P} \cdot Q_{j}}{\left\|X_{j}-X_{P}\right\|^{2}}, f\left(X_{j}\right)>f\left(X_{P}\right)
\end{array}, i, j=1, \cdots, m\right.
$$

Birbil uses the above measures to avoid premature convergence of the algorithm, and is primarily based on the consideration that although any non-current optimal particle can be used as a perturbation particle, the particle $X_{P}$ farthest from the current best particle $X_{\text {best }}$ is attracted by $X_{\text {best }}$ with minimal attraction particles do not cause a great deal of disturbance to the whole.

\section{RESEARCH ON CHAOS ELECTROMAGNETIC MECHANISM FUSION ALGORITHM}

Research shows that the optimization algorithms learn from each other's better optimization mechanisms to enrich the search behavior, which can greatly improve the global optimization ability [6-8]. In this paper, chaos optimization method is introduced in the electromagnetic-like mechanism algorithm. It is through the complementarities between the excellent optimization mechanisms to improve the global optimization effect, and provides a model that can be used for the organic fusion of electromagnetic-like algorithms and other algorithms.
The modified EM algorithm regards the particle $X_{P}$ farthest from the current optimal particle $X_{\text {best }}$ as the disturbing particle. In fact, only the most easily overlooked area in the previous calculation process is re-searched. Strictly speaking, it cannot traverse the entire area solution space. Considering the chaotic motion has the characteristics of randomness and periodicity [9], Logistic chaotic maps are introduced to generate chaotic variables when EM algorithm is used for optimization. This gives some disturbances to the current best particles and makes these particles to other areas in the feasible domain better areas move to achieve a global search.

Give the value of variable $S$ in generation $t$, value of generation $t+1$ can be obtained,

$$
S_{k}^{t+1}=\mu \cdot S_{k}^{t}\left(1-S_{k}^{t}\right), \quad 0<S_{k}<1, \quad k=1,2, \cdots, n
$$

In equation (10), $k$ is the number of chaotic variables, $t$ is chaos optimization iterations, and $\mu$ is the attractor. A small change in the initial value of a chaotic variable can lead to a significant change in its long-term behavior. The variable trajectory can traverse the entire search space.

EM algorithm is mainly used for global search, and chaos local search (Chaos Local Search, CLS) algorithm can chaos optimization of its results, that is, by improving the periodicity of spatial search to further improve the diversity of the population, to avoid falling into a local optimum. The specific steps of the CLS algorithm are as follows,

Step1. Let $t=0$, map the global extremer $X_{k}^{t}(k=1,2, \ldots, n)$ which found by the EM algorithm to the initial value of the chaotic variable $S_{k}^{t}$ between 0 and 1 according to formula (8).

$$
S_{k}^{t}=\left(X_{k}^{t}-l_{k}\right) /\left(X_{k}^{t}-u_{k}\right)
$$

In equation (11), the search upper and lower bounds of the $k$ dimension variable are still denoted by $u_{k}$ and $l_{k}$.

Step2. Calculate the chaotic variables of the next iteration

$$
S_{k}^{t+1}=4 S_{k}^{t}\left(1-S_{k}^{t}\right), \quad k=1,2, \ldots, n
$$

Step3. According to the following formula, convert $S_{k}^{t+1}$ to $X_{k}^{t+1}$

$$
X_{k}^{t+1}=l_{k}+S_{k}^{t+1}\left(u_{k}-l_{k}\right), \quad k=1,2, \ldots, n
$$


Step4. Calculate the objective function value corresponding to the new solution according to $X_{k}^{t+1}$. If the chaos search result is better than the global optimal solution vector $X^{0}=\left[X_{1}^{0}, X_{2}^{0}, \ldots, X_{n}^{0}\right]$ obtained by the previous EM algorithm, or the chaos search has reached the maximum number of iterations N2, the new searched better solution will be obtained. As a search result for CLS, otherwise set $t=t+1$ and go to Step2.

\section{Algorithm Performance Test And Comparative ANALYSIS}

To test the performance of the CEM fusion algorithm, the following four typical high-dimensional test functions are selected for the performance comparison of PSO, EM, and CEM algorithms,

F1: Sphere function

$$
f_{1}(x)=\sum_{i=1}^{n} x_{i}^{2} \quad-100 \leq x_{i} \leq 100
$$

The unimodal function, reaches the minimum value of 0 at $x_{i}=0$.

F2: Ackley function

$$
f_{2}(x)=-20 \exp \left(-0.2 \sqrt{\frac{1}{n} \sum_{i=1}^{n} x_{i}^{2}}\right)-\exp \left[\frac{1}{n} \sum_{i=1}^{n} \cos \left(2 \pi x_{i}\right)\right]+20+e \quad-30 \leq x_{i} \leq 30
$$

The multi-modal function is modulated by a cosine wave into a series of peaks or valleys in a flat region. The local minima are evenly distributed throughout the search space. It is used to test the ability of the algorithm to jump out of a local optimum. At $x_{i}=0$, it reaches a minimum value of 0 .

F3: Rosenbrock function

$$
f_{3}(x)=\sum_{i=1}^{n-1}\left[100\left(x_{i+1}-x_{i}^{2}\right)^{2}+\left(x_{i}-1\right)^{2}\right] \quad-30 \leq x_{i} \leq 30
$$

F4: Griewank function

$$
f_{4}(x)=\frac{1}{4000} \sum_{i=1}^{n} x_{i}^{2}-\prod_{i=1}^{n} \cos \left(\frac{x_{i}}{\sqrt{i}}\right)+1 \quad-100 \leq x_{i} \leq 100
$$

The multi-modal functions with significant mutual influence in each dimension, with infinitely many extreme points, are used to comprehensively examine the balance of the global and local search capabilities of the optimization algorithm, and the global minimum value 0 is taken at $x_{i}=0$.

The test process is implemented by MATLAB programming. To facilitate the comparative analysis of the algorithm performance, the parameters are set as follows, function dimension $n=80$, population size $n=100$, EM algorithm maximum iterations $N_{1}=2000$, local search times $L I S T=50$, chaotic search maximum times $N_{2}=1000$, each test repeat Perform 20 times.

Table 1 lists the Function (test function) and the corresponding $f_{\text {Global }}$ (the currently known global optimal solution), $f_{\text {Best }}$ (the optimal value of the algorithm running multiple times), $\bar{f}_{\text {Best }}$ (the optimal solution mean of the

\begin{tabular}{|c|c|c|c|c|c|c|c|c|c|c|}
\hline \multirow[b]{2}{*}{ Function } & \multicolumn{3}{|c|}{ PSO } & \multicolumn{3}{|c|}{ EM } & \multicolumn{3}{|c|}{ CEM } & \multirow[b]{2}{*}{$f_{\text {Global }}$} \\
\hline & $f_{\text {Best }}$ & $\bar{f}_{\text {Best }}$ & Time $(s)$ & $f_{\text {Best }}$ & $\bar{f}_{\text {Best }}$ & Time $(s)$ & $f_{\text {Best }}$ & $\bar{f}_{\text {Best }}$ & Time $(s)$ & \\
\hline Sphere & $8.75 e-5$ & 2.6829 & 63.5 & $7.12 \mathrm{e}-4$ & 1.5986 & 87.3 & $3.52 e-6$ & 0.2465 & 59.7 & 0 \\
\hline Ackley & $4.62 \mathrm{e}-3$ & 1.1534 & 172.8 & $5.86 e-3$ & 0.9265 & 149.2 & $9.83 e-4$ & 0.7523 & 108.4 & 0 \\
\hline Rosenbrock & $9.21 \mathrm{e}-4$ & 3.8675 & 168.2 & $2.70 e-3$ & 4.1239 & 136.8 & $5.47 \mathrm{e}-4$ & 1.9385 & 96.3 & 0 \\
\hline Griewank & $6.84 \mathrm{e}-3$ & 0.2736 & 97.3 & $8.45 e-3$ & 0.8357 & 105.6 & 7.85e-5 & 0.6193 & 75.2 & 0 \\
\hline
\end{tabular}
algorithm running multiple times), the data result of the Time (Algorithm Runtime).

TABLE I. CONTRAST AND ANALYSIS OF PSO, EM AND CEM TEST RESULTS

According to the analysis, for the easier convergence of the standard test function $\mathrm{F} 1$, the average optimal solution $\bar{f}_{\text {Best }}$, the global optimal solution $f_{\text {Best }}$, and the convergence speed (characterized by Time) of the CEM algorithm are significantly better than those of the PSO algorithm and the EM algorithm; the remaining few are more difficult to converge functions, CEM algorithms also exhibit better search performance than PSO and EM algorithms. Compared with the standard EM algorithm, the CEM fusion algorithm applies an external perturbation to some progeny particles through the embedded
Logistic chaos search, thus preventing the premature convergence of the algorithm.

The data in Table 1 shows that CEM can effectively improve the global optimization ability while basically not affecting the computational efficiency, and especially shows great advantages in jumping out of the local optimal solution.

\section{SUMMARY}

For these four test functions, the commonality is although the initial population is different, CEM and EM tend to be 
similar and converge faster than EM, especially in the latter part of the algorithm, and the efficiency of CEM is derived from It can jump out of the local convergence domain in time, and quickly move from the current optimal position to a better position, thus improving the speed of approaching the global optimal solution. Therefore, chaos mechanism is used to perform local refinement search to achieve global optimization of the EM algorithm get more ideal results.

The performance of EM and PSO is similar, and the improved EM algorithm that introduces chaos mechanism, namely CEM algorithm, is improved in both the accuracy of the solution and the convergence efficiency, and it is helpful to solve the problem of high-dimensional function optimization. At the same time, it is found that the better initial population is of great significance for speeding up the optimization. Therefore, there is still room for improvement in the performance of the CEM algorithm. The initial fitness can be optimized through multiple initial population selections, and the elite solution can be set to increase the initial fitness.

\section{ACKNOWLEDGEMENTS}

Thanks for support of the following fund projects,

[1] 'Thirteen Five' Jiangsu Province, a subject key provincial construction discipline project (2016-0802 Mechanical Engineering)

[2] Jiangsu university brand professional construction project funded projects (PPZY2015C251 mechanical design and manufacture and automation)

\section{REFERENCES}

[1] Li Xiaolei. A new intelligent optimization method-artificial fish school algorithm [D]. Hangzhou: Zhejiang University doctoral dissertation, 2003

[2] Birbil S.I., Shu-cherng, Fang. An electromagnetism-like mechanism for global optimization[J]. Journal of Global Optimization, 2003,25(3): 263-282

[3] Birbil S.I., FangS.C., SheuR.L. On the convergence of a population-based global optimization algorithm[J]. Journal of Global Optimization, 2004,30(2): 301-318

[4] GAO Liang, WANG Xiao-Juan, WEI Wei, CHEN Ya-Ya. An Improved Electromagnetic Mechanism Algorithm[J]. Journal of Huazhong University of Science and Technology(Natural Science), 2006,34(11): 4-6

[5] WANG Xiaojuan, GAO Liang, CHEN Asia. Electromagnetic mechanism and its application[J]. Applied Research of Computers, 2006,(6): 67-70.

[6] Hoai An Le Thi, Dinh Tao Pham, Nguyen V.Thoai. Combination Between Global and Local Methods for Solving an Optimization problem over the Efficient set. European Journal of Operational Research, 2002, 142:258-270

[7] Xian Liu, Wilsun Xu. A New Filled Function Applied to Global Ooptimization. Computer \& Operations Research, 2004, 31:61-80

[8] Peiliang Xu. A Hybrid Global Optimization Method: The Multi-dimensional Case. Journal of Computer and Applied mathematics, 2003, 155: 423-446

[9] Wu Tiebin, Cheng Yun, Zhou Taoyun, Yue Zhou. PID parameter optimization based on chaotic genetic algorithm[J]. Computer Simulation, 2009, 26(5): 202-205 